AperTO - Archivio Istituzionale Open Access dell'Università di Torino

\title{
UbiquiTO: a Multi-Device Adaptive Guide
}

\section{This is the author's manuscript}

Original Citation:

Availability:

This version is available http://hdl.handle.net/2318/89732

since 2018-03-08T14:33:16Z

Publisher:

Springer

Published version:

DOI:10.1007/978-3-540-28637-0_47

Terms of use:

Open Access

Anyone can freely access the full text of works made available as "Open Access". Works made available under a Creative Commons license can be used according to the terms and conditions of said license. Use of all other works requires consent of the right holder (author or publisher) if not exempted from copyright protection by the applicable law. 


\title{
UbiquiTO: a Multi-Device Adaptive Guide
}

\author{
Ilaria Amendola ${ }^{1}$, Federica Cena $^{1(2)}$, Luca Console ${ }^{2}$, Andrea Crevola ${ }^{1}$, Cristina \\ Gena $^{2}$, Anna Goy ${ }^{2}$, Sonia Modeo ${ }^{1(2)}$, Monica Perrero ${ }^{1}$, Ilaria Torre ${ }^{2}$, Andrea Toso ${ }^{1}$ \\ ${ }^{1}$ CSP innovazione nelle ICT s.c. a r.l., \\ Torino, Italy \\ \{amendola, cena, crevola, modeo, perrero, toso\}@csp.it \\ ${ }^{2}$ Dip. Di Informatica, Università di Torino \\ Torino, Italy \\ \{lconsole, cgena, goy, ilatorre\}@di.unito.it
}

\begin{abstract}
This paper describes UbiquiTO, an adaptive tourist guide, conceived as a "journey companion" for mobile users in Turin, aimed, for the current prototype, at supporting mobile workers helping them to organize their late afternoon and evening in town. The paper is intended to emphasize the most relevant feature of the system, that is the integration of different adaptation strategies in order to allow high flexibility in terms of device used, localization technology, user preferences and context conditions.
\end{abstract}

\section{Introduction}

The convergence of pervasive computers and communication networks offers new opportunities and challenges for systems designers and the exponential diffusion of devices such as PDAs and smart phones drives in the same direction, but the opportunity to use and profit from digital services depends on the possibility of adaptation to the mobile context, including input/output modalities, goals and location of the user, and so on. In particular, we think that the last feature represents a real add-on to common digital services, enabling the so-called location based services. Moreover, in order to make mobile services really useful and profitable, the characteristics and preferences of the users should be taken into account.

In this paper we describe UbiquiTO, an agent-based expert tourist guide for mobile users (the first prototype focuses on mobile workers), filtering the information and delivering it in the most appropriate way, depending on different factors: (a) Tourist services are provided according to the location of the user. (b) The user interface adapts to different types of devices; in the current prototype, we focused on PC and PDA. (c) The system exploits user profiles, including her interests, preferences and her previous visits to Turin, in order to provide personalized suggestions. (d) The interaction is adapted taking into account a set of context parameters such as the time of the day, the fact that the user is moving, and so on. Moreover, services are provided in two different ways: as consequence of explicit request from the user, who asks for a specific support (e.g., to find a hotel or a restaurant, or to get information about events or places of interests); by proactive activation, when the system itself, in 
specific situations, depending on the adaptation strategies mentioned above, autonomously provides the user with tourist advices. In the following, Section 2 provides an overview of the system architecture; Section 3 presents some details about the different forms of adaptation; Section 4 concludes the paper and compares UbiquiTO to some related works.

\section{UbiquiTO Architecture}

UbiquiTO architecture includes four main agents: the Recommender exploits the personalization rules to suggest items tailored to the user preferences and location; the Presentation Adapter exploits the adaptation rules to adapt the presentation (e.g. descriptions) to the user preferences, the device characteristics, and the context; the Interaction Manager handles the dialog with the user: each dialog step corresponds to the generation of a XML object representing the personalized content of the page to be displayed; the UI Generator handles the application of XSL stylesheets that transform the XML object into the (X)HTML pages representing the User Interface (UI), taking into account the different characteristics of the devices and the context features.

The architecture also includes four specialized modules, which handle, respectively, the user profile, the model of the device, the information about the location and a model of the context (environment conditions). Each specialized module exploits specific features stored in two main databases: the users $D B$ and the places $D B$.

\section{Adaptation Strategies}

As several studies suggested (e.g., [3]), adaptation techniques can be effectively exploited to handle the interaction in mobile user interfaces. In the current prototype, two user interfaces have been designed: one for PC and one for PDA (see Figure 1). The system can generate an adaptive version (AV) and a non-adaptive version (NAV) of both user interfaces.

Adaptation of Content. In the AV, in order to suggest places to visit, restaurants, accommodations and so on, the Recommender assigns a score to each item and orders them. The computation of this score takes into account: (a) the user's interest in the category the item belongs to; (b) the proximity of the item to the user position, in case the user exploits a mobile device. In the NVA, the items are ranked only according to their popularity. Moreover, in the AV, when the system is asked to provide an item description, it adds a list of suggestions tailored to the user profile and the user location (see right-hand side of PC and PDA user interfaces in Figure 1).

Adaptation of the User Interface. The Presentation Adapter personalizes: (a) the amount of information displayed, according to the screen size of the user's device; (b) the font size and background colour, according to context conditions (e.g., time of the day, movement or not) and user features (age, possible vision impairments, etc.). 


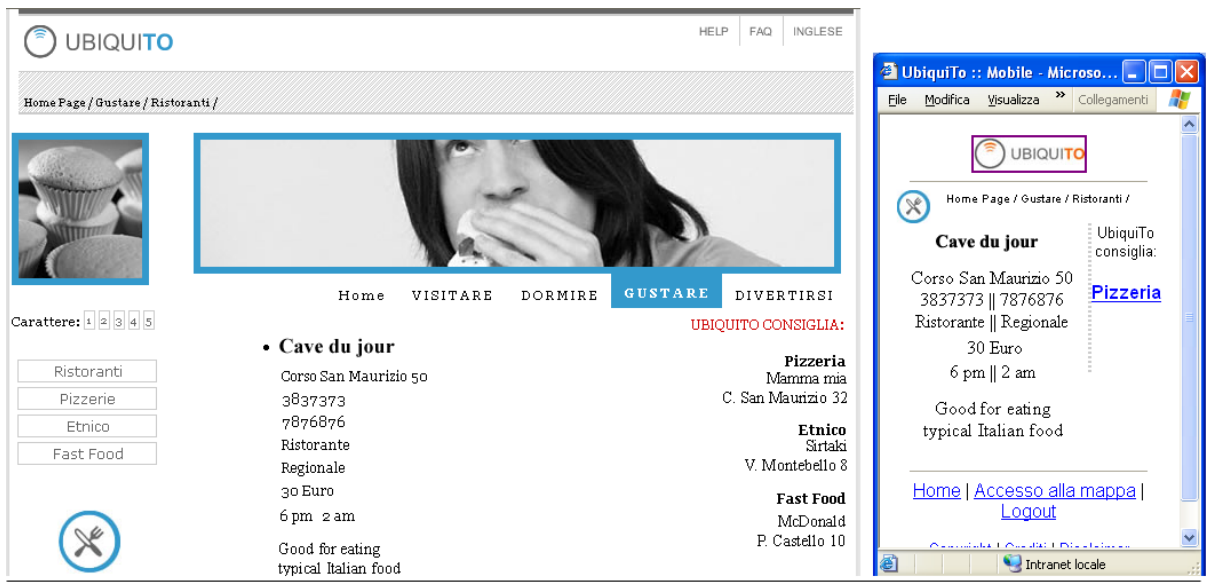

Fig. 1. UbiquiTO user interfaces: PC, on the left and PDA, on the right.

Localization Strategies. In the UbiquiTO project we considered four methods to localize the user and in the current prototype we implemented the first two ones:

1. User-driven localization. If the user is not equipped with a device supporting automatic positioning, like GPS, she has to provide the system with the coordinates of the point she is closest to. The UbiquiTO UI offers two different ways to specify the user's location: she can select a point from a list of items, or she can click on a sensitive map. The interaction with the map involves two steps, at different levels of detail: in the first step, the system shows the user a map representing the whole area (the center of Turin) and some of the most important points of interest in town (monuments, churches, etc.); in the second step, a more detailed map, representing a zoom of the previously selected area, is shown, containing a larger number of points of interest. In both cases, the user clicks on the point she is closest to, the system retrieves its coordinates from the places DB and computes the coordinates of the user position as approximately corresponding to those of the selected point. Notice that this form of localization may also be exploited by users to ask for information non current-location-dependent.

2. Wireless $L A N$. If the user mobile terminal is equipped with a WiFi receiver and enters in wireless modality, her position can be computed on the basis of the signals received from the different access points within the area. This positioning method is rather precise, but it can be exploited only within areas covered by wireless LAN. We have planned to test this kind of localization method inside the Environment Park (in Turin), in cooperation with INLAB (see [8]).

3. GPS. In this case the tourist's device contains a GPS receiver that enables the system to calculate the user position with great accuracy. Unfortunately, the GPS technology is affected by some limitations: its low diffusion; the slowness of the survey; the bad performance indoor.

4. Network-based positioning. Mobile networks operators can compute the user position quite precisely, on the basis of the distance of the user from the closest cell, even though the accuracy depends on the cells size (the smaller are the cells, the 
more accurate is the localization). Moreover, the main problem of this positioning method is represented by privacy restrictions, which in some countries, including Italy, prohibit telecom companies from releasing this kind of information.

Independently of the elicitation method used, the user position is represented by a couple of coordinates. Given this information, the system retrieves, from the places $\mathrm{DB}$, the coordinates of places to be recommended and calculates the distance between user position and every single place, in order to suggest the closest ones.

\section{Conclusions and related works}

In this paper we have presented UbiquiTO, an expert tourist guide for mobile users that adapts the content provided and the interaction to the user interest and physical location, as well as to the devices and context conditions. Mobile guides typically merge approaches from different fields. UbiquiTO borrows techniques from User Modelling and combines them with wireless technologies. The integration of different adaptation strategies is probably the most relevant aspect of UbiquiTO, since it supports a high flexibility, by adapting to heterogeneous factors. Several works in different areas are significantly related to the project. Even restricting the analysis to mobile guides, the number of systems developed since the first prototype, Cyberguide [1], is high (see Guide [4], Lol@ [11], Crumpet [10], Real [2], SmartKom [12], Deep Map [7], as a sample of the main ones). Therefore, we will take into consideration just those ones that are most comparable with the main features of our system.

As said, the most important characteristic of UbiquiTO is its flexibility, due to the integration of device and location adaptation with adaptation to the user features. This combination enables the tourist to use her own mobile terminal, not necessarily equipped with specific positioning devices or client-side applications, and to benefit from the advantages of the adaptation. From this point of view, Crumpet [10] is probably the most relevant related project. It personalizes services to the user's current location, interests, and history of interaction, on PDA and smart phones; it also adapts the presentation to changing technical environments and exploits GPS or other operator based technologies (e.g. GSM, UMTS) to localize the user. Guide [4] is another relevant project. It is a tourist guide for the city of Lancaster, adaptive to the location of the user, her walking speed, the places already visited, the time of the day and the language and interests of the user. The main lack of the system concerns the client device: Guide services may be accessed only using an ad hoc terminal rented at the Lancaster tourist office. Lol@ [11], a guide for the city of Vienna, is adaptive toward the device, but not toward the user features. It uses GPS as positioning technology and exploits a GIS system for the generation of the map. As in UbiquiTO, the layout is adapted using XSL stylesheets. In spite of its complex infrastructure, the lack of adaptivity toward the user interest, preferences and goals may compromise, as emphasized by [3], the possibility to enjoy the services in mobile environments. In term of flexibility, one last feature we would like to briefly discuss is the modality to elicit the user position. A good half of the outdoor systems uses GPS, while just a few allow the estimation of the position by interacting with the user. Guide, Lol@, Real 
and Deep Map are some examples of integration of alternative methods to elicit the user position [6]. As seen, one of the goals of UbiquiTO is to go in the same direction: the current prototype offers two modalities: layered maps and WiFi technology; in the next versions, we will experiment GPS modality and possibly Network-based solutions. A relevant aspect of the map is that it is built upon the concept of points of proximity which shares the principles of landmarks, already experimented in several contexts (see [9]). The main idea is that they represent relevant points in the user mental map and help the user to construct a mental representations of unfamiliar environments.

The first version of UbiquiTO is currently ready to be tested. We have planned a layered evaluation of the system, aimed at testing both the adaptation of the content and the adaptation of the user interface. Many aspects will be improved in future versions. Different mobile devices will be taken into account (e.g., smart phones, onboard equipments) and a larger set of services will be included. Moreover, in order to automatically update the user profiles, learning mechanisms will be studied and implemented. Finally, localization mechanism exploiting GPS will be experimented.

\section{References}

1. Abowd, D. A., Atkeson, C. G., Hong, J., Long, S., Pinkerton, M.: Cyberguide: A Mobile Context-Aware Tour Guide. Wireless Networks 3(5) (1996) 421-433

2. Baus, J., Kray, C., Kruger, A.: Visualization of route descriptions in a resource-adaptive navigation aid. Cognitive Processing 2(2-3) (2001) 323-345

3. Billsus, D., Brunk, C., Evans, C., Gladish., B., Pazzani, M.: Adaptive Interfaces for Ubiquitous Web Access. Comm. of the ACM 45(5) (2003) 34-38

4. Cheverest, K., Davies, N., Mitchel,1 K., Smyth, P.: Providing tailored context-aware information to city visitors. In Adaptive Hypermedia and Adaptive Web-Based Systems (AH 2000). LNCS Vol. 1892, Springer-Verlag, Berlin Heidelberg New York (2000) 73-85

6. Kray, C., Baus, J.: A survey of mobile guides. Workshop on HCI in mobile guides, 5th Int. Symposium on HCI with Mobile Devices and Services. Udine, Italy (2003)

7. Malaka, R. and Zipf, A.: DEEP MAP - Challenging IT research in the framework of a tourist information system. Information and Communication Technologies in Tourism. Proc. of ENTER 2000, Springer Computer Science, Wien New York (2000) 15-27

8. Marchioro, C.E., Ghittino, A., Ferri, D., Borri, R., Dovis, F.: Wireless Campus project. TERENA Networking Conference 2003. Zagreb, Croatia (2003)

9. Michon, P. E., Denis, M.: When and why are visual landmarks used in giving directions. In: Montello, D.R. (ed.): Spatial Information Theory. Lecture Notes in Computer Science, Vol. 2205, Springer-Verlag, Berlin Heidelberg New York (2001) 292-305

10. Poslad, S., Laamanen, H., Malaka, R., Nick, A., Buckle, P., Zipf, A.: CRUMPET: Creation of User-Friendly Mobile Services Personalised for Tourism. 3G Mobile Communication Technologies. London, UK (2001) 26-29

11. Pospischil, G., Umlauft, M., Michlmayr, E.: Designing LoL@, a Mobile Tourist Guide for UMTS. Information Technology \& Tourism 5(3), Cognizant Communication Corporation (2003) 151-164

12. Wahlster W., Reithinger N., Blocher A.: SmartKom: Multimodal Communication with a Life-Like Character. 7th European Conference of Speech Communication and Technology (Eurospeech 2001), Vol. 3, Aalborg, Denmark (2001) 1547-1550 